\title{
Retroalimentación negativa mediante reformulaciones correctivas: impacto en el desarrollo de la distinción modal en español como segunda lengua
}

\author{
Irene Alonso ApARICIO \\ Universidad de Granada \\ Rheinich-Westfälische Technische Hochschle, Aachen (Alemania)
}

Recibido: 3 junio 2009 / Aceptado: 9 octubre 2009

ISSN: $1697-7467$

\begin{abstract}
RESUMEN: Este artículo aborda sucintamente las posibilidades de la interacción oral y de la reformulación correctiva para contribuir al desarrollo de la interlengua. A continuación, se discute la adquisición de una estructura inherentemente difícil en la adquisición del español/segunda lengua (E/L2), la distinción modal. La segunda parte del trabajo presenta un estudio empírico cuyo objetivo es examinar la capacidad de las reformulaciones correctivas para contribuir al desarrollo de la distinción modal en oraciones condicionales potenciales en E/L2. Finalmente, se presenta una reflexión acerca de cómo pueden nuestros resultados informar sobre el proceso de enseñanza y aprendizaje de la lengua no nativa.
\end{abstract}

Palabras clave: interacción oral, retroalimentación negativa, reformulación correctiva, distinción modal.

Negative Feedback Through Corrective Recast: Impact in the Development of Mood Selection in Spanish as a Second Language

\begin{abstract}
The first part of this paper briefly deals with the possibilities of interaction and corrective recasts to facilitate interlanguage development. Following, we discuss the acquisition of an inherently difficult structure in Spanish/Second Language (S/L2), mood selection. In the second part of the paper, we report on a study that focuses on the possibilities of corrective recasts to aid the acquisition of mood selection in secondtype conditional sentences in S/L2. Conclusions are drawn about how our data might inform second language teaching and learning.
\end{abstract}

Key words: interaction, negative feedback, corrective recast, mood selection. 


\section{INTRODUCCIÓN}

Para quien decide abordar la tarea de aprender una segunda lengua (L2) ${ }^{1}$, la habilidad de comunicarse de manera fluida y correcta es posiblemente uno de los objetivos más anhelados. No obstante, el camino recorrido hacia la consecución de dicho objetivo no está exento de dificultades, siendo quizás una de las más notables la superación de los fallos en la comprensión y expresión oral cuando se participa en la conversación oral espontánea con hablantes nativos o con compañeros aprendices con un dominio de la L2 superior al propio.

Si nos situamos en el contexto formal, hasta hace relativamente poco tiempo y bajo el prisma del conductismo, los fallos han constituido lo indeseado y se ha tendido desde las aulas primero a prevenirlos, y en caso de originarse, a erradicarlos ipso facto (métodos audiolingual y situacional). Sin embargo, reflexión teórica y datos empíricos han puesto de manifiesto en las últimas décadas la viabilidad de muchos de estos fallos para evidenciar el progreso lingüístico del aprendiz (Corder, 1967), y más aún, para constituir un marco en el que el aprendizaje lingüístico siga desarrollándose (Long, 1983, 1996, 2007). Por lo tanto, volviendo al contexto de la comunicación oral, podríamos argüir que la interacción oral, pese a constituir un marco de fallos potenciales, se erige como posible escenario en el que la adquisición lingüística tenga lugar.

En las líneas que se suceden reflexionamos desde el paradigma cognitivo-interaccionista, sobre las posibilidades de la interacción oral para contribuir al desarrollo de la interlengua (IL) y sobre uno de los ajustes conversacionales ampliamente documentado en la literatura, las reformulaciones correctivas. A continuación, discutimos la adquisición de una estructura inherentemente difícil en la adquisición del español/L2 (E/L2), la distinción modal. En la segunda parte de este trabajo, presentamos un estudio empírico cuyo objetivo es examinar la capacidad de las reformulaciones correctivas para contribuir al desarrollo de la distinción modal en oraciones condicionales potenciales. Finalmente, concluimos con una reflexión acerca de cómo pueden nuestros resultados informar sobre el proceso de enseñanza y aprendizaje de la lengua no nativa.

\section{INTERACCIÓN ORAL Y REFORMULACIÓN CORRECTIVA}

Los fallos que se originan en la conversación oral entre el hablante nativo (HN) y el hablante no nativo (HNN) o entre HNN-HNN (siendo uno de los HNNs lingüísticamente más competente en la L2 que su interlocutor), pueden suponer un escollo para el desarrollo de la comunicación e incluso el abandono o el cambio de tema de conversación en el acto comunicativo. No obstante, estos fallos también pueden desencadenar la negociación de significado (Long, 1983), es decir, una serie de ajustes conversacionales que buscan salvar la comunicación (p. e., cuando ante la perplejidad de nuestro interlocutor reformulamos lo que acabamos

1. No establecemos diferencias entre los términos adquisición/aprendizaje y segunda lengua/lengua extranjera. Empleamos únicamente el término segunda lengua (L2) para referirnos a la lengua no nativa, y empleamos indistintamente los términos adquisición y aprendizaje para referirnos al proceso de desarrollo y dominio de una L2. 
de decir, o cuando ante la incomprensión del enunciado de nuestro interlocutor, le solicitamos una aclaración o repetición). Esta observación, junto con las conclusiones avanzadas en el campo de estudio de la adquisición de segundas lenguas (AL2), llevan a Long (1996) a postular que la negociación de significado que tiene lugar en la interacción oral no sólo salva o facilita la comunicación, sino que además facilita la adquisición en sí de la competencia lingüística (Hipótesis de la Interacción):

La negociación de significado, y especialmente la negociación que desencadena ajustes en la interacción por parte del hablante nativo o de un interlocutor más competente, facilita la adquisición porque conecta de forma productiva el input, las capacidades internas del aprendiz, una atención particularmente selectiva, y el output. (Long, 1996: 451-452) (Traducción de la autora -TA en adelante) $)^{2}$

En efecto, los estudios en AL2 han señalado como condiciones teóricamente importantes en la adquisición de una L2:

(1) La exposición a un input ${ }^{3}$ comprensible (Krashen, 1982; Long, 1983; cf. White, 1987).

(2) La oportunidad de producción lingüística (Swain, 1985, 1993, 1995, 1998), porque permite al aprendiz: (2.a) emplear significativamente sus propios recursos lingüísticos; (2.b) verse forzado a trasmitir su mensaje «con precisión, coherencia, y apropiación» (1985: 249) (TA) ${ }^{4}$ (output forzado o empujado); (2.c) reparar en lo que no sabe o sabe sólo parcialmente y verse en la necesidad de procesar sintácticamente un input posterior que le permita advertir los recursos formales de los que carece; (2.d) formular y comprobar hipótesis sobre la L2, e idílicamente reestructurar su IL; y, (2.e) fomentar la fluidez o automatización de conocimientos.

(3) La captación, o atención consciente subjetiva a las formas del input (Schmidt, 1990, 1994), y la captación de la diferencia entre la propia IL y la L2 (Schmidt, 1994), y entre lo que se quiere decir y lo que se es capaz de decir (Swain, 1985, 1993, 1995, 1998). Y,

(4) La retroalimentación negativa, o provisión de información sobre las opciones incorrectas de la lengua meta (White, 1987, 1989, 1991).

Considerando entonces estas condiciones teóricamente importantes en la adquisición de la L2, la Hipótesis de la Interacción postula que la negociación de significado facilita el desarrollo de la IL porque permite al aprendiz recibir un input más comprensible (p. e., cuando ante el gesto de perplejidad de su interlocutor no nativo, el HN reformula con otras palabras el sentido de su enunciación). Asimismo, la negociación de significado facilita el

2. «[...] negotiation for meaning, and especially negotiation work that triggers interactional adjustme nts by the NS or more competent interlocutor, facilitates acquisition because it connects input, internal learner capacities, particularly selective attention, and output in productive ways»».

3. Por su difusión en la literatura, empleamos las voces inglesas input, output, e intake, para referirnos respectivamente a caudal lingüístico, producción lingüística, y parte de datos percibida e incorporada en la memoria a corto plazo.

4. «[...] precisely, coherently, and appropriately». 
desarrollo de la IL porque proporciona al aprendiz la oportunidad de emplear significativamente sus propios recursos lingüísticos y en ocasiones le fuerza a producir un output más preciso, coherente, y apropiado (piénsese por ejemplo en la petición de aclaración que solicita el HN al HNN ante la incomprensión de su enunciado y la reformulación precisa y coherente que tiene que realizar el HNN para ser entendido). Por otro lado, los ajustes conversacionales pueden además advertir al HNN de sus limitaciones lingüísticas, y esto a su vez puede llevarle a buscar los recursos lingüísticos necesarios en muestras de lengua posteriores (un caso así sería el del aprendiz al que se le solicita una reformulación y cuando la va a llevar a cabo toma consciencia de su falta de recursos lingüísticos y de su necesidad de desarrollarlos). Finalmente, otra explotación viable de la negociación de significado es la provisión de retroalimentación negativa más o menos explícita, y por lo tanto, el favorecer que el HNN capte la diferencia entre su IL y la L2 (tal sería el caso del HN que reformula el enunciado gramaticalmente incorrecto de su interlocutor no nativo mientras que éste capta la retroalimentación contenida en ella).

En definitiva, aprovechando las palabras de Gass y Mackey (2007: 194), la interacción oral facilita el desarrollo de la IL:

[...] porque proporciona a los aprendices la oportunidad de recibir input modificado y de recibir retroalimentación, tanto explícitamente como implícitamente, lo que a su vez puede dirigir la atención de los estudiantes a aspectos problemáticos de sus interlenguas y a forzarles a producir un output modificado. (TA $)^{5}$

Pues bien, en el estudio de cómo facilita la interacción oral el desarrollo de la IL, uno de los ajustes conversacionales ampliamente documentado en la literatura es la reformulación o reformulación correctiva. Esta expresión alude a la reproducción que realiza el HN (o el HNN con mayor dominio de la L2) de la locución gramaticalmente incorrecta del HNN, corrigiendo los aspectos gramaticalmente incorrectos, pero sin alterar el significado de la intención comunicativa. Por lo tanto, la reformulación correctiva proporciona al HNN retroalimentación negativa incidental (no deliberada) e implícita mientras ambos interlocutores centran su atención en el significado del intercambio comunicativo. La idoneidad de este ajuste conversacional radica entonces, en el hecho de que el HNN puede centrar su atención en la forma meta y captar la diferencia entre ésta y su propia IL sin preocuparse por entender su significado, dado que supone una reproducción de su propio mensaje.

Si bien teóricamente las posibilidades de esta estrategia conversacional son evidentes, la investigación de su impacto en el desarrollo de la IL no está exenta de dificultades, dada la complejidad que supone determinar qué datos evidencian la captación y el aprendizaje (Gass y Mackey, 2007). Es decir, si por ejemplo un HN reformula la locución gramaticalmente incorrecta de su interlocutor no nativo y éste la reproduce total o parcialmente, ¿imita simplemente la reformulación sin comprender por qué?, ¿la reproduce como estrategia para mostrar que efectivamente ha comprendido la retroalimentación negativa contenida en ella?, ¿la repite

5. «[...] as it provides learners with opportunities to receive modified input and to receive feedback, both explicitly and implicitly, which in turn may draw learners' attention to problematic aspects of their interlanguages and push them to produce modified output». 
conscientemente a la vez que la compara con la estructura que él hubiera utilizado para trasmitir el mismo contenido? Pese a esta dificultad metodológica, Long (2007) ha identificado recientemente en la literatura hasta 60 estudios descriptivos, quasi-descriptivos, y experimentales, acerca de la existencia y utilidad de las reformulaciones en el contexto formal y natural. Tras una revisión parcial pero representativa de la muestra empírica que emplea instrumentos de recogida de datos fiables (pre-test/post-test, juicios introspectivos y retrospectivos, etc.), Long concluye, en primer lugar, que las reformulaciones existen en una frecuencia relativamente alta en el contexto formal y natural. En segundo lugar, Long concluye que los aprendices captan la retroalimentación negativa contenida en ellas, y que ésta es además utilizable y (en ocasiones) utilizada. Y por último, expone Long que si bien no son necesarias para la adquisición, las reformulaciones correctivas sí que parecen facilitarla.

\section{LA ADQUISICIÓN DEL SUBJUNTIVO EN ESPAÑOL/SEGUNDA LENGUA}

La distinción modal entre indicativo y subjuntivo es un área que entraña gran complejidad y le cuesta dominar al aprendiz de español/L2 (E/L2) pese a una considerable exposición y práctica significativa en el contexto formal y/o natural. La dificultad de aprendizaje de este aspecto formal se atribuye al modo subjuntivo debido a, entre otros factores: (a) su inexistencia en otras lenguas; (b) la amplitud de casos en los que se utiliza; (c) su (frecuente) relación con la subordinación (sintaxis compleja); y, (d) su dificultad de procesamiento porque: (d.1) en ocasiones implica sólo un cambio vocálico en la desinencia verbal (canto-cante); (d.2) en algunas estructuras es comunicativamente redundante (p. e., en Dudo que coma mucho, ¿por qué procesar la a de coma cuando Dudo comunica duda?); y, (d.3) generalmente aparece en la posición media del enunciado, una posición que según la investigación en procesamiento de la información, es menos susceptible de ser procesada que las posiciones inicial o final ( $\mathrm{Vid}$. VanPatten, 1996).

Collentine $(1995,2003)$ arguye que la adquisición de la distinción modal está sujeta al desarrollo psicolingüístico del aprendiz, concretamente a su capacidad para interpretar y producir sintaxis compleja. En este sentido, Collentine (1995) muestra que los aprendices de nivel intermedio, tras 4 semestres de instrucción, poseen una base lingüística insuficiente como para generar sintaxis compleja y por lo tanto, para beneficiarse de la instrucción dirigida a la distinción modal (si el estudiante es incapaz de generar una oración subordinada, ¿cómo le vamos a pedir que seleccione el modo correspondiente?). Asimismo, la investigación concluye que en las fases iniciales de la adquisición, la forma del subjuntivo pasa desapercibida por las razones previamente expuestas, y los matices semánticos propios del subjuntivo no llaman la atención del aprendiz hasta una fase intermedia de desarrollo de la IL (Vid. Collentine, 2003). Otra línea de investigación que aborda el desarrollo del subjuntivo en E/L2 se centra en el impacto que sobre él tienen distintas metodologías. Es decir, más que documentar el desarrollo en sí del subjuntivo, esta línea busca dilucidar beneficios de la instrucción, generalmente oponiendo metodologías inspiradas en el procesamiento del input frente a metodologías centradas en la producción lingüística significativa. Los resultados de esta investigación sugieren que ambas propuestas metodológicas pueden ser igualmente eficaces en su desarrollo (Alonso Aparicio, 2007; Collentine, 1998; Farley, 2000; Llopis García, 2007). 


\section{ESTUdio de LA AdQUiSICIÓN DEL PRETÉRITO IMPERFECTO DE SUBJUNTIVO EN ORACIONES CONDICIONALES POTENCIALES POR ESTUDIANTES NORTEAMERICA- NOS}

\subsection{Motivación y descripción}

La revisión de la literatura que hemos llevado a cabo motiva la presente investigación con el fin de documentar la capacidad de la reformulación correctiva para contribuir al desarrollo de una forma inherentemente difícil como es el uso del pretérito imperfecto de subjuntivo en las oraciones condicionales potenciales en E/L2. Asimismo, nos llama la atención la ausencia de estudios que hayan documentado el desarrollo de esta forma, es decir, no tenemos constancia de que exista estudio alguno centrado en el desarrollo del pretérito imperfecto de subjuntivo, más bien la investigación parece haberse centrado en el desarrollo del presente de subjuntivo en participantes que o bien desconocían la forma, o bien conocían su uso en contextos diferentes al del tratamiento de instrucción. Por último, nos sorprende la ausencia de procedimientos de instrucción implícitos en la investigación centrada en el impacto de distintas metodologías para el desarrollo de esta forma, más bien, la naturaleza de estos procedimientos ha sido explícita, generalmente incluyendo explicaciones metalingüísticas. Esta última observación tiene lógica dada la dificultad de esta forma y especialmente en los casos en los que se presenta la forma/regla meta por vez primera.

Por lo tanto, considerando la literatura revisada, proponemos la siguiente cuestión para investigación: ¿Permite al aprendiz la provisión de abundante retroalimentación negativa mediante reformulaciones correctivas captar y entender el uso del pretérito imperfecto de subjuntivo en las oraciones condicionales potenciales? Abordamos esta cuestión en el estudio que se presenta a continuación.

\subsection{El pretérito imperfecto de subjuntivo en oraciones condicionales potenciales}

Este estudio aborda el desarrollo del pretérito imperfecto de subjuntivo en las oraciones condicionales potenciales. Su elección está motivada por las dificultades que los participantes del estudio venían demostrando en su uso, empleando sistemáticamente el presente de indicativo o el condicional en la oración subordinada en algunos casos, y empleando indistintamente casi cualquier tiempo verbal en otros casos. No obstante, cabe mencionar que todos los participantes dominaban la morfología del subjuntivo en presente y pretérito imperfecto, y estaban familiarizados con su uso en otros contextos.

Tradicionalmente se define a las oraciones condicionales como aquéllas en las que el cumplimiento de lo enunciado en la oración principal depende de la realización de lo enunciado en la oración subordinada (cf. Montolío, 1999). Tomando como criterio el carácter de la condición expresada en la oración subordinada, las oraciones condicionales se clasifican en: (a) oraciones reales o verosímiles -la realización de la condición es factible; (b) oraciones potenciales -la realización de la acción es dudosa o poco factible; y, (c) oraciones irreales la realización de la acción es inviable:

(a) Si hace buen tiempo mañana, saldré a dar un paseo.

(b) Si las condiciones laborales fueran mejores, cambiaría de ciudad.

(c) Si hubiera empezado antes, ya habría terminado. 
Si bien el nexo típico para introducir la oración condicional es $s i$, a menudo se usan otras conjunciones como cuando y como, y locuciones conjuntivas como a no ser que, con tal (de) $q u e$, etc. No obstante, nuestro estudio centra su atención exclusivamente en la estructura de la oración condicional potencial introducida por si. En cuanto a dicha estructura, la secuencia sintáctica prototípica de estas oraciones es: $s i+$ pretérito imperfecto de subjuntivo + condicional simple (b) (cf. Montolío, 1999).

\subsection{Participantes}

La muestra de participantes de este estudio está constituida por un total de 13 estudiantes norteamericanos (procedentes de California) que se encontraba participando en un programa de intercambio con la Universidad de Granada en el momento de la investigación. La totalidad de los sujetos, con edades comprendidas entre los 20 y 21 años, se ofreció voluntariamente a participar en el estudio y llevaba residiendo en nuestro país 6 meses. La mayoría de los sujetos tenía experiencia en el estudio de otras lenguas extranjeras, y en el caso concreto del E/L2, existía en su estudio una variación intragrupal considerable, siendo 3 años la duración mínima para algunos sujetos (presumiblemente coincidente con la educación universitaria), y 12 años la duración máxima para 2 sujetos (posiblemente un estudio iniciado en la educación primaria como L3 ó L4). Sin embargo, pese a esta variación intragrupal en el tiempo de estudio del español, la totalidad de la muestra demostró en el pre-test una actuación muy pobre en la selección modal en las oraciones condicionales potenciales (no obstante, como ya se ha expuesto, todos los participantes dominaban la morfología del subjuntivo en presente y pretérito imperfecto y estaban familiarizados con su uso en otros contextos). El nivel de los participantes se determinó por tanto intermedio-alto o avanzado (equivalente al nivel B2 ó C1 del Marco Común Europeo de Referencia).

Para nuestro estudio, los participantes fueron asignados a dos grupos de trabajo: un grupo reformulación que recibió abundante retroalimentación negativa mediante reformulaciones correctivas en la interacción oral $(\mathrm{n}=7)$, y un grupo interacción que no recibió retroalimentación negativa en la interacción oral $(\mathrm{n}=6)$. Dentro de cada grupo se establecieron 3 subgrupos que trabajaron con la investigadora de forma individual (grupo $1(\mathrm{n}=2)$, grupo $2(\mathrm{n}=2)$ y grupo $3(\mathrm{n}=3)$ para el grupo reformulación, y grupo $1(\mathrm{n}=2)$, grupo $2(\mathrm{n}=2)$, y grupo $3(\mathrm{n}=2)$ para el grupo interacción).

\subsection{Procedimientos y materiales de instrucción}

Este estudio se desarrolló a lo largo de 4 sesiones de trabajo: pre-test (sesión 1/día 1); primera sesión de clase (sesión 2/día 2); segunda sesión de clase (sesión 3/día 3); y, post-test (sesión 4/día 4). El periodo de instrucción para cada subgrupo de trabajo abarcó por tanto 2 sesiones de clase de aproximadamente 30 minutos cada una (una sesión por día) en las que los 6 subgrupos realizaron las mismas 2 tareas (una tarea por día). Como se ha mencionado

6. Nótese que esta secuencia puede expresar además la noción de irrealidad cuando su referencia temporal es simultánea al momento en el que se está produciendo el discurso (Si estuviera ahora en Valencia, me iría a comer una paella a la Albufera) (Vid.Montolío, 1999). No obstante, excluimos esta posibilidad de nuestro estudio. 
antes, la diferencia entre los grupos de trabajo residió en que los participantes de los 3 subgrupos del grupo reformulación recibieron abundante retroalimentación negativa mediante reformulaciones correctivas en la realización de las tareas, mientras que los participantes de los 3 subgrupos del grupo interacción no recibieron ninguna retroalimentación negativa en la realización de las tareas.

En cuanto a las tareas, éstas consistieron en la adaptación del juego de mesa La Oca a los contextos temáticos «¿En qué situación 'harías’ X?» (Día 1) y «A mi chico/a le “concedería' X si Y» (Día 2) (Vid. Apéndice A). Las reglas del juego se asemejaron a las del juego original, teniendo los jugadores que avanzar desde la casilla «salida» hasta la casilla «meta», con posibilidad de pérdida de turno, retroceso de casilla, e incluso retroceso a la casilla «salida». Cada casilla representaba un número y cada número remitía a una tarjeta que presentaba una situación hipotética. La investigadora participó en el juego como una jugadora más.

\subsection{Prueba experimental}

La prueba experimental que ha servido de base para este estudio consiste en un test administrado un día antes (pre-test) y un día después (post-test) de las sesiones de clase. Este test estuvo constituido por dos tareas escritas, una de interpretación y otra de producción, y una entrevista oral grabada (Vid. Apéndice B).

En cuanto a las tareas escritas, la tarea de interpretación consistió en un juicio de gramaticalidad que presentaba un total de 62 oraciones de las que sólo 10 medían el conocimiento de la forma meta y las 52 restantes servían de función distractora para evitar que el estudiante reparara en ella y aplicara su conocimiento metalingüístico. El cometido del estudiante fue decidir la gramaticalidad o agramaticalidad de las oraciones, y en el caso de detectar agramaticalidad, subrayar la parte afectada. De las 10 oraciones que medían el conocimiento del ítem lingüístico meta, se insertaron un $50 \%$ gramaticalmente correctas y un $50 \%$ gramaticalmente incorrectas. Por su parte, la tarea de producción consistió en completar una batería de 64 oraciones conjugando los verbos que se ofrecían entre paréntesis. De nuevo, sólo 10 de estas 64 oraciones se consideraron para el estudio, sirviendo las 54 oraciones restantes de función distractora. Asimismo, si bien se administró la misma versión del test como pretest y post-test, en el post-test se modificaron las oraciones que servían de función distractora en ambas tareas y se cambió el orden de las oraciones empleadas para la recogida de datos con respecto a la versión del pre-test. En cuanto al tiempo de realización de las tareas, para evitar en la medida de lo posible la aplicación de conocimiento metalingüístico, sometimos a los estudiantes a presión de tiempo. Concretamente, los tiempos asignados fueron: 10 minutos para la tarea de interpretación y 13 minutos para la tarea de producción. Estos tiempos se determinaron tomando como referencia los tiempos empleados por 2 hablantes nativos en la realización del test ( 7 minutos para la tarea de interpretación y 10 para la de producción) y aumentándolos en 3 minutos para compensar demoras en la comprensión lectora. En cuanto a la puntuación, en la tarea de interpretación se asignó un punto a cada oración gramatical correctamente aceptada y a cada oración agramatical correctamente subrayada. En la tarea de producción, se asignó un punto a cada verbo correctamente conjugado.

Con respecto a la entrevista oral grabada, ésta se diseñó con el fin de documentar la producción lingüística espontánea del aprendiz y consistió en la simulación de una entrevista 
de trabajo. Habiéndose debatido previo a la entrevista y de forma personal, qué futuro laboral anhelaba cada participante, la investigadora representó el papel de empresaria y el participante de candidato potencial para ocupar un puesto en la empresa anhelada. Esta prueba incluyó un total de 11 preguntas de las que las 5 primeras recogieron información personal del candidato y sirvieron de función distractora, las 5 siguientes plantearon 5 situaciones hipotéticas que invitaban al uso de la forma meta, y la última se empleó para cerrar la entrevista y servir asimismo de función distractora. En cuanto a las preguntas que plantearon situaciones hipotéticas, incluyeron a su vez dos preguntas, una primera de respuesta cerrada si/no que planteaba la situación hipotética, y una segunda que sólo tenía razón de ser si se había contestado la primera en afirmativo. Esta segunda pregunta siempre se planteó en los términos ¿en qué circunstancias? y fue la que invitó al uso de la forma meta. Por lo tanto, para el análisis de los datos de la entrevista oral, sólo se consideraron las respuestas a estas segundas preguntas. Asimismo, cabe mencionar que aunque la segunda pregunta ¿en qué circunstancias? podía responderse con una única circunstancia, para el análisis se consideraron todas las circunstancias que cada participante estimara oportuno plantear. Finalmente, en cuanto a la puntuación, a la totalidad de los contextos planteados por cada estudiante se asignó una valoración del $100 \%$, a cada forma verbal correctamente producida en cada circunstancia hipotética se asignó un punto, y el porcentaje de aciertos se calculó entonces considerando el número de formas correctas con respecto a la totalidad de contextos creados.

\subsection{Resultados}

\subsubsection{Tarea de interpretación}

La tabla 1 muestra la comparación de medias pre-test (PR)/post-test (PO) en la tarea de interpretación para los 2 grupos de trabajo. En un rango 10, los datos muestran un ascenso de la media $(\mu)$ de 4.28 (pre-test) a 9.71 (pos-test) para el grupo reformulación (GR), y de 4.33 (pre-test) a 9.50 (post-test) para el grupo interacción (GI).

Tabla 1: Comparación de medias pre-test/post-test en la tarea de interpretación.

\begin{tabular}{|l|c|c|}
\hline & Pre-test & Post-test \\
\hline Grupo reformulación $(G R)(n=7)$ & 4.28 & 9.71 \\
\hline Grupo interacción $(G I)(n=6)$ & 4.33 & 9.50 \\
\hline
\end{tabular}

En cuando a la significación de este ascenso, la tabla 2 presenta los resultados de la prueba de muestras relacionadas en la tarea de interpretación para los 2 grupos en el pre-test (PR) y el post-test (PO). Si para el GR se plantea como $\mathrm{H}_{0}: \mu($ GR-PR $)=\mu\left(\right.$ GR-PO) e $\mathrm{H}_{1}$ : $\mu($ GR-PR $) \neq \mu\left(\right.$ GR-PO), y para el GI se plantea como $\mathrm{H}_{0}: \mu(\mathrm{GI}-\mathrm{PR})=\mu(\mathrm{GI}-\mathrm{PO})$ e $\mathrm{H}_{1}: \mu$ $(\mathrm{GI}-\mathrm{PR}) \neq \mu$ (GI-PO), dado que en ambos casos $p=0.000$, es decir, $p \leq 0.05$, se rechaza la hipótesis nula $\left(\mathrm{H}_{0}\right)$ y se acepta la hipótesis alternativa $\left(\mathrm{H}_{1}\right)$. Luego, se puede afirmar que las puntuaciones obtenidas en el PO por ambos grupos son significativamente distintas a las obtenidas en el PR. 
Tabla 2: Prueba de muestras relacionadas en la tarea de interpretación para ambos grupos en el pre-test y post-test.

\begin{tabular}{|c|c|c|c|c|c|c|c|c|c|}
\hline & \multicolumn{5}{|c|}{ Diferencias relacionadas } & \multirow[b]{3}{*}{$\mathrm{t}$} & \multirow[b]{3}{*}{ gl } & \multirow{3}{*}{$\begin{array}{l}\text { Sig. } \\
\text { (bil) }\end{array}$} \\
\hline & & \multirow[b]{2}{*}{ Media } & \multirow{2}{*}{$\begin{array}{l}\text { Des. } \\
\text { típ. }\end{array}$} & \multirow{2}{*}{$\begin{array}{l}\text { Error } \\
\text { típ. de } \\
\text { media }\end{array}$} & \multicolumn{2}{|c|}{$\begin{array}{l}95 \% \text { Int. confianza } \\
\text { para la diferencia }\end{array}$} & & & \\
\hline & & & & & Inferior & Superior & & & \\
\hline GE-PR/ & GE-PO & -5.42 & 1.39 & .52 & -6.72 & -4.13 & -10.27 & 6 & .000 \\
\hline GI-PR/ & GI-PO & -5.16 & 1.32 & .54 & -6.56 & -3.77 & -9.52 & 5 & .000 \\
\hline
\end{tabular}

En cuanto a la diferencia de medias entre los grupos en el post-test, la tabla 1 indica una diferencia intergrupal de 0.21 del GR sobre el GI. En cuanto a la significación de esta diferencia, si planteamos como $\mathrm{H}_{0}: \mu(\mathrm{GR}-\mathrm{PO})=\mu(\mathrm{GI}-\mathrm{PO})$ e $\mathrm{H}_{1}: \mu$ (GR-PO) $\neq \mu$ (GI-PO), dado que la tabla 3 muestra que mediante la prueba de Levene se asumen varianzas iguales (siendo $\mathrm{H}_{0}: \sigma^{2}(\mathrm{GR}-\mathrm{PO})=\sigma^{2}$ (GI-PO) e $\mathrm{H}_{1:} \sigma^{2}$ (GR-PO) $\neq \sigma^{2}$ (GI-PO), dado que $p=0.17$, es decir, $p$ $>0.05$, se acepta $\mathrm{H}_{0}$ ), la significación para el test de medias es de $p=0.21$, es decir, $p>$ 0.05. Por lo tanto, se acepta $\mathrm{H}_{0}: \mu(\mathrm{GR}-\mathrm{PO})=\mu(\mathrm{GI}-\mathrm{PO})$ y se asume que no hay diferencias significativas entre las notas obtenidas por el GR y por el GI en el PO.

Tabla 3: Prueba de muestras independientes en la tarea de interpretación para ambos grupos en el post-test.

\begin{tabular}{|c|c|c|c|c|c|c|c|c|c|c|}
\hline & \multicolumn{2}{|c|}{$\begin{array}{l}\text { Prueba de } \\
\text { Levene para la } \\
\text { igualdad de } \\
\text { varianzas }\end{array}$} & \multicolumn{7}{|c|}{ Prueba $T$ para la igualdad de medias } \\
\hline & & \multirow[b]{2}{*}{$\mathrm{F}$} & \multirow[b]{2}{*}{ Sig. } & \multirow[b]{2}{*}{$\mathrm{T}$} & \multirow[b]{2}{*}{ Gl } & \multirow{2}{*}{$\begin{array}{l}\text { Sig. } \\
\text { (bil) }\end{array}$} & \multirow{2}{*}{$\begin{array}{c}\text { Dif. } \\
\text { de } \\
\text { med } \\
\text { ias }\end{array}$} & \multirow{2}{*}{$\begin{array}{l}\text { Erro } \\
\text { r típ. } \\
\text { de } \\
\text { dif. }\end{array}$} & \multicolumn{2}{|c|}{$\begin{array}{c}95 \% \text { Intervalo } \\
\text { confianza para dif }\end{array}$} \\
\hline & & & & & & & & & Inf. & Sup \\
\hline $\begin{array}{l}\mathrm{P} \\
\mathrm{O} \\
\mathrm{S} \\
\mathrm{T}\end{array}$ & $\begin{array}{c}\text { Se han } \\
\text { asumido } \\
\text { varianzas } \\
\text { iguales }\end{array}$ & 2.16 & .17 & .57 & 11 & .57 & .21 & .37 & -.60 & 1.03 \\
\hline $\begin{array}{l}\mathrm{T} \\
\mathrm{E} \\
\mathrm{S} \\
\mathrm{T} \\
\end{array}$ & $\begin{array}{c}\text { No se han } \\
\text { asumido } \\
\text { varianzas } \\
\text { iguales } \\
\end{array}$ & & & .55 & 7.78 & .59 & .21 & .38 & -.68 & 1.11 \\
\hline
\end{tabular}

\subsubsection{Tarea de producción}

En la tabla 4 se muestra la comparación de medias pre-test $(\mathrm{PR}) /$ post-test $(\mathrm{PO})$ en la tarea de producción para los 2 grupos de trabajo. En un rango 10, los datos muestran un ascenso de la media $(\mu)$ de 1.42 (pre-test) a 9.57 (pos-test) para el grupo reformulación (GR), y de 1.66 (pre-test) a 9.66 (post-test) para el grupo interacción (GI). 
Tabla 4: Comparación de medias pre-test/post-test en la tarea de producción.

\begin{tabular}{|l|c|c|}
\hline & Pre-test & Post-test \\
\hline Grupo reformulación $(n=7)$ & 1.42 & 9.57 \\
\hline Grupo interacción $(n=6)$ & 1.66 & 9.66 \\
\hline
\end{tabular}

En cuanto a la significación de este ascenso, la tabla 5 presenta los resultados de la prueba de muestras relacionadas. Si para el GR se plantea como $\mathrm{H}_{0}: \mu(\mathrm{GR}-\mathrm{PR})=\mu(\mathrm{GR}-\mathrm{PO})$ e $\mathrm{H}_{1}: \mu$ (GR-PR) $\neq \mu$ (GR-PO), y para el GI se plantea como $\mathrm{H}_{0}: \mu(\mathrm{GI}-\mathrm{PR})=\mu(\mathrm{GI}-\mathrm{PO})$ e $\mathrm{H}_{1}: \mu(\mathrm{GI}-\mathrm{PR}) \neq \mu$ (GI-PO), dado que en ambos casos $p=0.000$, es decir, $p \leq 0$ '05, se rechaza $\mathrm{H}_{0}$ y se acepta $\mathrm{H}_{1}$. Por lo tanto, se puede afirmar que las puntuaciones obtenidas en el PO por ambos grupos son significativamente distintas a las obtenidas en el PR.

Tabla 5: Prueba de muestras relacionadas en la tarea de producción para ambos grupos en el pre-test y post-test.

\begin{tabular}{|c|c|c|c|c|c|c|c|c|c|}
\hline & \multicolumn{5}{|c|}{ Diferencias relacionadas } & \multirow[b]{3}{*}{$\mathrm{t}$} & \multirow[b]{3}{*}{ Gl } & \multirow{3}{*}{$\begin{array}{l}\text { Sig. } \\
\text { (bil) }\end{array}$} \\
\hline & & \multirow[b]{2}{*}{ Media } & \multirow{2}{*}{$\begin{array}{c}\text { Des. } \\
\text { típ. }\end{array}$} & \multirow{2}{*}{$\begin{array}{l}\text { Error } \\
\text { típ. de } \\
\text { media }\end{array}$} & \multicolumn{2}{|c|}{$\begin{array}{c}95 \% \text { Int. De confianza } \\
\text { para la diferencia }\end{array}$} & & & \\
\hline & & & & & Inferior & Superior & & & \\
\hline GE-PR/ & GE-PO & -8.14 & 2.11 & .80 & -10.10 & -6.18 & -10.18 & 6 & .000 \\
\hline GI-PR/ & GI-PO & -8.00 & 1.78 & .73 & -9.87 & -6.12 & -10.95 & 5 & .000 \\
\hline
\end{tabular}

En cuanto a la diferencia de medias entre los grupos en el PO, la tabla 4 indica una diferencia intergrupal de 0.9 puntos del GI sobre el GR. En cuanto a la significación de esta diferencia, si planteamos como $\mathrm{H}_{0}: \mu(\mathrm{GR}-\mathrm{PO})=\mu(\mathrm{GI}-\mathrm{PO})$ e $\mathrm{H}_{1}: \mu(\mathrm{GR}-\mathrm{PO}) \neq \mu$ (GI-PO), dado que la tabla 6 muestra que mediante la prueba de Levene se asumen varianzas iguales (considerando $\mathrm{H}_{0}: \sigma^{2}(\mathrm{GR}-\mathrm{PO})=\sigma^{2}(\mathrm{GI}-\mathrm{PO})$ e $\mathrm{H}_{1:} \sigma^{2}(\mathrm{GR}-\mathrm{PO}) \neq \sigma^{2}$ (GI-PO), dado que $p=$ 0.54 , es decir, $p>0.05$, se acepta $\mathrm{H}_{0}$ ), la significación para el test de medias es de $p=0.75$, es decir, $p>0.05$. Luego, se acepta $\mathrm{H}_{0}: \mu$ (GR-PO) $=\mu$ (GI-PO) y se asume que no hay diferencias significativas entre las notas obtenidas por el GR y por el GI en el PO.

Tabla 6: Prueba de muestras independientes en la tarea de producción para ambos grupos en el post-test.

\begin{tabular}{|c|c|c|c|c|c|c|c|c|c|c|}
\hline & \multicolumn{2}{|c|}{$\begin{array}{c}\text { Prueba Levene para } \\
\text { igualdad varianzas }\end{array}$} & \multicolumn{7}{|c|}{ Prueba T para la igualdad de medias } \\
\hline & & \multirow[b]{2}{*}{$\mathrm{F}$} & \multirow[b]{2}{*}{ Sig. } & \multirow[b]{2}{*}{$\mathrm{T}$} & \multirow[b]{2}{*}{ Gl } & \multirow{2}{*}{$\begin{array}{l}\text { Sig. } \\
\text { (bil) }\end{array}$} & \multirow{2}{*}{$\begin{array}{c}\text { Dif. } \\
\text { de } \\
\text { med } \\
\text { ias }\end{array}$} & \multirow{2}{*}{$\begin{array}{l}\text { Erro } \\
\text { r típ. } \\
\text { de } \\
\text { dif. }\end{array}$} & \multicolumn{2}{|c|}{$\begin{array}{l}\text { 95\% Int. } \\
\text { confianza } \\
\text { dif. }\end{array}$} \\
\hline & & & & & & & & & Inf. & Sup \\
\hline $\begin{array}{l}\mathrm{P} \\
\mathrm{O} \\
\mathrm{S} \\
\mathrm{T}\end{array}$ & $\begin{array}{c}\text { Se han } \\
\text { asumido } \\
\text { varianzas } \\
\text { iguales }\end{array}$ & .39 & .54 & -.32 & 11 & .75 & -.09 & .29 & -.74 & .55 \\
\hline $\begin{array}{l}\mathrm{T} \\
\mathrm{E} \\
\mathrm{S} \\
\mathrm{T}\end{array}$ & $\begin{array}{c}\text { No se han } \\
\text { asumido } \\
\text { varianzas } \\
\text { iguales }\end{array}$ & & & -.32 & 10.80 & .75 & -.09 & .29 & -.73 & .55 \\
\hline
\end{tabular}




\subsubsection{Entrevista oral}

La tabla 7 muestra la comparación de medias pre-test (PR)/post-test (PO) en la entrevista oral para los 2 grupos de trabajo. En un rango 100\%, los datos muestran un ascenso de la media $(\mu)$ de $0 \%$ (pre-test) a $74.90 \%$ (post-test) para el grupo reformulación (GR), y de $0 \%$ (pre-test) a 78.05\% (post-test) para el grupo interacción (GI). En cuanto a la diferencia de medias entre los grupos en el PO, se observa una diferencia intergrupal de 3.15\% del GI sobre el GR.

Tabla 7: Comparación de medias pre-test/post-test en la entrevista oral.

\begin{tabular}{|l|c|c|}
\hline & Pre-test & Post-test \\
\hline Grupo reformulación $(G R)(n=7)$ & $0 \%$ & $74.90 \%$ \\
\hline Grupo interacción $(G I)(n=6)$ & $0 \%$ & $78.05 \%$ \\
\hline
\end{tabular}

No obstante, dado que el número de contextos creados para emplear la forma meta estaba sujeto a la voluntad de cada estudiante, el análisis de las transcripciones produjo la dificultad de determinar qué estudiante presentaba mayor dominio de la forma y la lengua meta en general, si aquel que arriesga produciendo un discurso elaborado con 15 contextos hipotéticos (traducido en 4 páginas de trascripción) y por lo tanto corre el riesgo de equivocarse (Vid. Infra Tabla 8, Grupo Interacción, Estudiante 2), o aquel que produce un discurso pobre, con 4 contextos hipotéticos (traducido en 1 página de trascripción) y tiene por lo tanto menor riesgo de equivocarse (Vid. Infra Tabla 8, Grupo Reformulación, Estudiante 1). Por esta razón, no hemos llevado a cabo un análisis estadístico de los resultados y hemos considerado más oportuno dejarlos para un análisis futuro que considere el resto de competencias.

Tabla 8: Comparación individual de medias pre-test/post-test en la entrevista oral.

\begin{tabular}{|l|c|c|l|c|c|}
\hline \multicolumn{3}{|c|}{ Grupo Reformulación } & \multicolumn{3}{c|}{ Grupo Interacción } \\
\hline EE & Pre-test & Post-test & EE & Pre-test & Post-test \\
\hline E 1 & $0 / 10=\mathbf{0 \%}$ & $4 / 4=\mathbf{1 0 0} \%$ & E 1 & $0 / 4=\mathbf{0 \%}$ & $4 / 6=\mathbf{6 6 . 6 6 \%}$ \\
\hline E 2 & $0 / 7=\mathbf{0 \%}$ & $5 / 10=\mathbf{5 0 \%}$ & E 2 & $0 / 8=\mathbf{0 \%}$ & $9 / 15=\mathbf{6 0} \%$ \\
\hline E 3 & $0 / 10=\mathbf{0} \%$ & $6 / 8=\mathbf{7 5 \%}$ & E 3 & $0 / 3=\mathbf{0 \%}$ & $3 / 3=\mathbf{1 0 0 \%}$ \\
\hline E 4 & $0 / 9=\mathbf{0 \%}$ & $6 / 7=\mathbf{8 5 . 7 1 \%}$ & E 4 & $0 / 4=\mathbf{0 \%}$ & $4 / 4=\mathbf{1 0 0 \%}$ \\
\hline E 5 & $0 / 5=\mathbf{0 \%}$ & $7 / 11=\mathbf{6 3 . 6 3 \%}$ & E 5 & $0 / 7=\mathbf{0 \%}$ & $7 / 11=\mathbf{6 3 . 6 3 \%}$ \\
\hline E 6 & $0 / 7=\mathbf{0 \%}$ & $5 / 10=\mathbf{5 0 \%}$ & E 6 & Invalidado & Invalidado \\
\hline E 7 & $0 / 8=\mathbf{0 \%}$ & $5 / 5=\mathbf{1 0 0} \%$ & & & \\
\hline Media & $\mathbf{0 \%}$ & $\mathbf{7 4 . 9 0 \%}$ & Media & $\mathbf{0 \%}$ & $\mathbf{7 8 . 0 5 \%}$ \\
\hline
\end{tabular}

EE: Estudiantes

E: Estudiante 


\section{CONCLUSIÓN Y REFLEXIÓN}

Sintetizando el análisis de datos que acabamos de presentar, podemos concluir que ambas propuestas de instrucción, con y sin reformulaciones correctivas, han resultado eficaces en la promoción de la forma meta. Sin embargo, no se han encontrado diferencias significativas entre el comportamiento de los estudiantes asignados al grupo reformulación y aquéllos asignados al grupo interacción. Por lo tanto, respondiendo a nuestra pregunta de investigación, sí podemos afirmar que la provisión de abundante retroalimentación negativa mediante reformulaciones correctivas permite al aprendiz captar y entender el uso del pretérito imperfecto de subjuntivo en las oraciones condicionales potenciales. No obstante, al menos considerando nuestro estudio, no podemos concluir que la retroalimentación negativa haya sido el desencadenante de la adquisición, pues ha producido los mismos resultados que la interacción desprovista de ella.

Una explicación potencial a los resultados obtenidos la encontramos en la naturaleza de las tareas realizadas: en grupos reducidos (máximo 3 alumnos por grupo), sistemáticas, y motivadoras. Dado que el conjunto de jugadores se contabilizó en máximo 4 participantes, los turnos de juego de los estudiantes estuvieron muy próximos a los de la investigadora, quien al participar como jugadora proporcionaba muestras correctas de la forma meta. Asimismo, la adaptación de nuestro juego de mesa ( $L a O C a$ ) se llevó a cabo con un recorrido de aproximadamente 40 casillas, luego todos los estudiantes dispusieron de sobradas ocasiones para practicar la forma meta. Finalmente, la temática y el diseño de ambas tareas se revelaron bastante motivadores y provocaron que los estudiantes se implicaran a consciencia en el juego (quizás por tratarse de una pseudo-competición y sin duda porque la temática les resultó de interés).

Otra posible explicación a nuestros resultados radica en el nivel de desarrollo lingüístico de la muestra $(\mathrm{B} 2 / \mathrm{C} 1)$. El hecho de que nuestros participantes presentaran un relativo dominio de la distinción modal en la subordinación nominal e incluso adverbial, posiblemente sirvió de trampolín para que la exposición reiterada a la forma meta desencadenara la captación y entendimiento de la distinción modal en las oraciones condicionales potenciales. Es decir, el conocimiento previo de los alumnos sobre la forma y uso del subjuntivo en otros contextos, pareció ser determinante para que el estudiante que no obtuvo retroalimentación negativa extendiera su conocimiento sobre el uso del subjuntivo a un contexto diferente (dedujera otra regla de uso del subjuntivo). En este sentido, nuestros datos se encuentran en consonancia con la propuesta de que la instrucción deba ajustarse al nivel de desarrollo psicolingüístico del aprendiz (Pienemann 1984), y más concretamente, con la conclusión de Collentine (1995) de que los efectos de la instrucción sobre la distinción modal se perciben una vez que el estudiante ha alcanzado una fase de desarrollo «sintáctica» o avanzada.

De nuestros resultados se pueden extraer algunas consideraciones que podrían trasladarse al aula de segundas lenguas. En primer lugar, cabe mencionar la importancia de diseñar cuidadosamente tareas que se ajusten al nivel de desarrollo psicolingüístico del aprendiz, que fomenten la interacción en grupos reducidos, y que resulten motivadoras. Asimismo, frente a la creencia arraigada de gran parte de docentes y discentes sobre la importancia y necesidad de la corrección del error, siempre que el tratamiento se ajuste al desarrollo psicolingüístico del aprendiz, la exposición en la interacción a muestras correctas de la lengua meta puede 
bastar para un desarrollo óptimo de la forma meta. En este sentido, la provisión de retroalimentación negativa proactiva -previa al error- mediante explicaciones metalingüísticas con mayor o menor grado de explicitud formal, puede asimismo resultar redundante.

Finalmente y como prospectiva investigadora, cabe replicar nuestro estudio con sujetos experimentales que no posean conocimiento de la existencia de la distinción modal en español. Es decir, cabe plantearse si contribuirán de igual modo la exposición a la lengua meta y la reformulación correctiva a la captación y entendimiento del uso del pretérito imperfecto de subjuntivo en las oraciones condicionales potenciales en el caso de no tener los aprendices conocimiento del modo subjuntivo. Asimismo, otra posible línea de investigación puede abordar las posibilidades de la reformulación correctiva para prevenir la fosilización de formas desviadas de la norma estándar en el caso de la distinción modal. Por último, podría resultar también interesante estudiar el impacto de la reformulación correctiva para contribuir al desarrollo de la distinción modal en español como lengua materna.

\section{ReFERENCIAS}

Alonso Aparicio, I. (2007). «La instrucción basada en el procesamiento frente al enfoque ecléctico para el aprendizaje del subjuntivo en la enseñanza de E/LE», en Porta Linguarum, 8: 4162.

Collentine, J. (1995). «The Development of Complex Syntax and Mood-Selection Abilities by Intermediate-level Learners of Spanish», en Hispania, 78, 3: 123-136.

Collentine, J. (1998). «Processing Instruction and the subjunctive», en Hispania, 81, 3: 576-587.

Collentine, J. (2003). «The Development of Subjunctive and Complex-Syntactic Abilities among FL Spanish Learners», en B. Lafford y R. Salaberry (eds.), Studies in Spanish Second Language Acquisition: the State of the Science. Washington, DC: Georgetown University Press, 74-97.

Corder, S. P. (1967). «The Significance of Learner's Errors», en IRAL, 5: 161- 170.

Farley, A. P. (2000). The relative effects of processing instruction and meaning-based output instruction on L2 acquisition of the Spanish subjunctive. University of Illinois at UrbanaChampaign (tesis doctoral inédita).

Gass, S. y Mackey, A. (2007). «Input, Interaction, and Output in Second Language Acquisition», en B. VanPatten y J. Williams (eds.), Theories in Second Language Acquisition. An Introduction. Mahwah: Lawrence Erlbaum, 175- 199.

Krashen, S. (1982). Principles and Practice in Second Language Acquisition. London: Prentice Hall International.

Long, M. (1983). Native speaker/non-native speaker conversation and the negotiation of comprehensible input», en Applied Linguistics, 4, 2: 126-141.

Long, M. (1996). «The Role of the Linguistic Environment in Second Language Acquisition», en W. Rithie y T. Bhatia (eds.), Handbook of Second Language Acquisition. San Diego: Academic Press, 413-468.

Long, M. (2007). Problems in SLA. New Jersey: Lawrence Erlbaum Associates.

Llopis García, R. (2007). «Procesamiento del input y mejora en el output para el aprendizaje de segundas lenguas: un estudio basado en la Instrucción de Procesamiento para la enseñanza del subjuntivo español», en Revista Nebrija de Lingüística Aplicada a la Enseñanza de Lenguas, 1, 1: 100-123. 
Montolío, E. (1999). «Las construcciones condicionales», en I. Bosque y V. Demonte, Gramática descriptiva de la lengua española. Madrid: Espasa-Calpe: 3643-3738.

Pienemann, M. (1984). «Psychological Constraints on the Teachability of Languages», en Studies in Second Language Acquisition, 6, 2: 186- 214.

Schmidt, R. (1990). «The role of consciousness in second language learning», en Applied Linguistics, $11,129-158$.

Schmidt, R. (1994). «Deconstructing consciousness in search of useful definitions for applied linguistics», en AILA Review, 11: 11-26.

Swain, M. (1985). «Communicative competence: some roles of comprehensible input and comprehensible output in its development», en S. Gass y C. Madden (eds.), Input in second language acquisition. Rowley, MA: Newbury House, 235-256.

Swain, M. (1993). «The Output Hypothesis: Just Speaking and Writing Aren't Enough», en The Canadian Modern Language Review, 50, 1: 158-164.

Swain, M. (1995). «Three functions of output in second language learning», en G. Cook y B. Seidlhofer (eds.), Principle and practice in applied linguistics: Studies in honour of H.G. Widdowson. Oxford: Oxford University Press, 125-144.

Swain, M. (1998). «Focus on form through conscious reflection», en C. Doughty y J. Williams (eds.), Focus on form in classroom second language acquisition. Cambridge: Cambridge University Press, 64-81.

Vanpatten, B. (1996). Input processing and grammar instruction: Theory and research. Westport, CT: Ablex.

White, L. (1987). «Against comprehensible input: the input hypothesis and the development of second language competence», en Applied Linguistics, 8: 95-110.

White, L. (1989). «The principle of adjacency in second language acquisition: Do L2 learners observe the subset principle?», en S. Gass y J. Schachter (eds.), Linguistic perspectives on second language acquisition. Cambridge: Cambridge University Press, 134-158.

White, L. (1991). «Adverb placement in second language acquisition: some effects of positive and negative evidence in the classroom», en Second Language Research, 7: 133-161. 


\section{APÉNDICE A}

Muestra adaptada de las tareas

Día 1: ¿En qué situación 'harías' $X$ ? (Muestra de tarjetas incluidas)

1. ¿En qué situación declinarías una oferta de trabajo magnífica?

2. ¿En qué situación abandonarías tus proyectos de fututo?

3. ¿En qué situación visitarías un país en guerra?

4. ¿En qué situación te dejarías seducir por una persona que no te atrae?

5. ¿En qué situación no volarías en avión?

Día 2: A mi chico/a... (Muestra de tarjetas incluidas)

1. Le presentaría a mis padres si...

2. Le llevaría a París si...

3. Le sugeriría un cambio de look si...

4. Le propondría vivir juntos si...

5. Le mentiría si...

\section{APÉNDICE B \\ Muestra adaptada de la prueba experimental}

\section{Tarea de interpretación}

Decide si las siguientes oraciones son o no correctas. Si piensas que alguna oración es incorrecta, ¡no la corrijas!, sólo subrava la parte de la oración que es incorrecta.

1. ¡Oh, no! Está lloviendo y he olvidado la paraguas en casa. (Función distractora)

2. Si tuviera algo más de dinero, me plantearía comprar el coche de tu padre.

3. Javier le regaló un reloj con incrustaciones de piedras preciosos. (Función distractora)

4. No sé a qué hora llegaré, pero te llamaré al móvil en cuanto llego. (Función distractora)

5. No sabría qué contestar si me preguntaría por ella.

\section{Tarea de producción}

Completa las siguientes oraciones con la forma correcta del verbo que aparece entre paréntesis.

1. ¿Un sueño? Si (poder/yo), eliminaría el hambre.

2. No voy a instalar ningún programa de música en el ordenador hasta que no lo (formatear/yo). (Función distractora)

3. A: Estoy bastante harta, todavía no (comprar/yo) los regalos de reyes. (Función distractora) 
B: Y, ¿a qué

A: A inspirarme. Si lo

4. ¿Sabías que la risa (Función distractora)

5. Como no Plaza de España. (Función distractora)

\section{Guión para la entrevista oral: Entrevista de trabajo}

\section{Cuéntame algo sobre ti:}

1. ¿Qué experiencia tienes en este campo en particular?

2. ¿Estás buscando trabajo en otras empresas también?

3. ¿Por qué quieres trabajar con nosotros?

4. ¿Trabajas bien en equipo?

5. ¿Cuál dirías que es tu punto más fuerte?, y ¿tu punto más débil?

\section{Vamos a ver ahora cómo reaccionarías en algunas circunstancias hipotéticas}

1. ¿Estarías dispuesto/a a trabajar por la noche, horas extras o fines de semana? En caso afirmativo, ¿en qué circunstancias?

2. ¿Estarías dispuesto/a a mudarte por el trabajo? En caso afirmativo, ¿en qué circunstancias?

3. ¿Estarías dispuesto/a a poner los intereses de la empresa por encima de los tuyos? En caso afirmativo, ¿en qué circunstancias?

4. ¿Mentirías a tu jefe? En caso afirmativo, ¿en qué circunstancias?

5. ¿Le comunicarías a tu jefe una acción deshonesta de un compañero/a de trabajo? En caso afirmativo, ¿en qué circunstancias?

\section{Finalmente:}

1. ¿Por qué deberíamos contratarte? 